\title{
Hybrid Recognition for One Stroke Style Cursive Handwriting Characters
}

\author{
Teng Long and Lian-Wen Jin \\ School of Electronics and Information \\ South China University of Technology, Guangzhou 510641, P.R.China
}

\begin{abstract}
On-line handwriting recognition has continued to persist as a popular research field while pen computing applications are widely used in recent years. This paper proposes a novel hybrid system for one stroke style cursive handwriting character recognition. In the system, user can use fingertip to write various kinds of virtual characters (represented by trajectory of fingertip) such as alpha-numeric characters and Chinese characters through a digital camera based user interface. Without pen-up and pen-down information, the virtual characters are written in one stroke. An on-line and an off-line recognition method for such kind of cursive characters are proposed. A hybrid approach of these two methods is proposed to combine the advantages of both of them. Benefit from the integration, the recognition accuracy was increased from $80.6 \%$ (off-line classifier) and $83.4 \%$ (on-line classifier) to $90.9 \%$ (integrated) for stroke order free one stroke cursive handwriting Chinese characters.
\end{abstract}

\section{Introduction}

On-line handwriting character recognition has been researched for more than 40 years [1][2]. It provides a natural and convenient way for human-machine interaction. Due to the effort of researchers around the world, great advances of on-line handwriting recognition technology were made in the last two decades[1]. The current systems are already able to recognize regular script with very high accuracy. However, the recognition of cursive characters still remains unsolved [2]. Because it is more often and naturally for people to write characters in a cursive style especially when fast input is needed, the recognition of handwritten cursive characters gained particular interest in state-of-the-art character recognition research [6][16][17][19][20].

In this paper, we propose a novel hybrid system for one stroke (unistroke) style cursive handwriting character recognition which is shown in Fig. 1(a). In this system, user can use the fingertip to write various kinds of virtual characters (represented by trajectories of fingertip, without any ink information) such as alpha-numeric characters and Chinese characters through a digital camera based user interface. Compared with most pen input devices, camera input devices occupy much less space and can also provide big enough area for user to write characters intuitively [18], whereas it is not naturally for user to tell the system pen-up or pen-down information when he writes in the air by fingertip. Therefore, virtual characters are written in one stroke. Some examples of virtual characters are shown in Fig. 1(b). Unlike strokes connected characters written by pen-tip, a virtual character can be written with radicals overlaid, i.e., each radical may be written independent of its normal spatial relationship to the rest of the character. In the second row, the Chinese character “案” is written with radicals overlaid, this kind of writing style combines advantages of easier finger writing and better recognition efficiency as smaller range movement of fingertip is needed and radicals can be written comparatively bigger and explicitly in a small low resolution area.

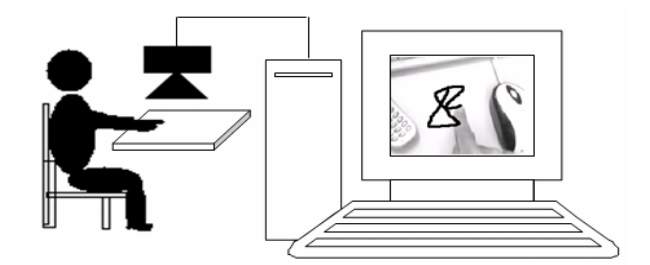

(a)

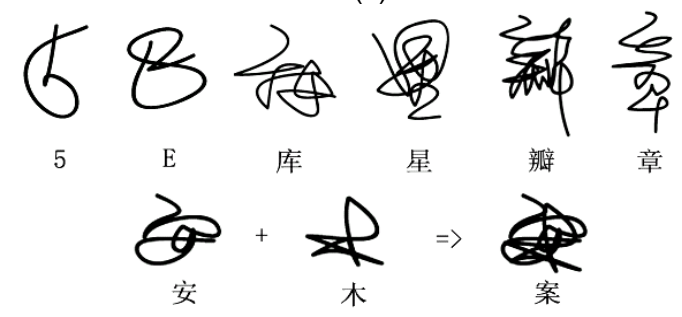

(b)

Fig. 1. The proposed system for finger-writing character input. (a) illustration of the system; (b) some examples of one stroke cursive (virtual) characters 
The recognition of such kind of virtual characters poses a new challenge in handwriting recognition area. Some researchers have proposed recognition approaches which are robust to strokes connection (e.g. [3]), whereas few recognition methods are tested on full strokes connected test set. Kiyota et al. [4] discussed the recognition of characters with partial strokes overlaid which are written by blindfolded person, but the proposed approach is writer dependent, and strokes are not fully connected. Shimodaira et al. [5] proposed their recognition system for overlaid characters, but the context information is needed.

In this paper, a hybrid system for one stroke cursive character recognition is proposed. In this system, an on-line handwritten character recognition approach is employed for recognizing cursive distorted virtual characters which are strokes connected and may have strokes or radicals overlaid. Based on dynamic time warping algorithm (DTW) [15], the on-line recognizer can effectively cope with large variations in stroke shape and position. However, it is stroke order sensitive. In order to add the capability of recognition for stroke order free Chinese characters, an off-line handwritten character recognizer is combined in the system. Benefit from the integration of on-line and off-line recognition techniques, the system can recognize stroke order free one stroke cursive characters. The promising experimental results demonstrate that the proposed hybrid system can recognize one stroke cursive characters with a high level of accuracy $(>90 \%)$.

\section{System overview}

Our vision based recognition system employs a digital camera for tracking user's fingertip. A block diagram of the system's structure is illustrated in Fig. 2. When user moves the fingertip to write virtual characters, the location of fingertip in each frame is find out and recorded. When a character is finished written, the trajectory of fingertip is restored by connecting the recorded points of fingertip in successive frames. After being filtered and deleted wild points, the preprocessed trajectory is similar to a handwritten one stroke character by pen-tip. The on-line and off-line recognizers integrated in the system are designed for such kind of characters. The final recognition result among possible candidates from the two classifiers is selected by an integrator.

The dashed line connecting off-line and on-line classifier in Fig. 2 provides a coarse classification by off-line recognizer is optional for user to select. If selected, it can speed up the online recognition process, however characters with radicals overlaid may not be recognized correctly in this way.

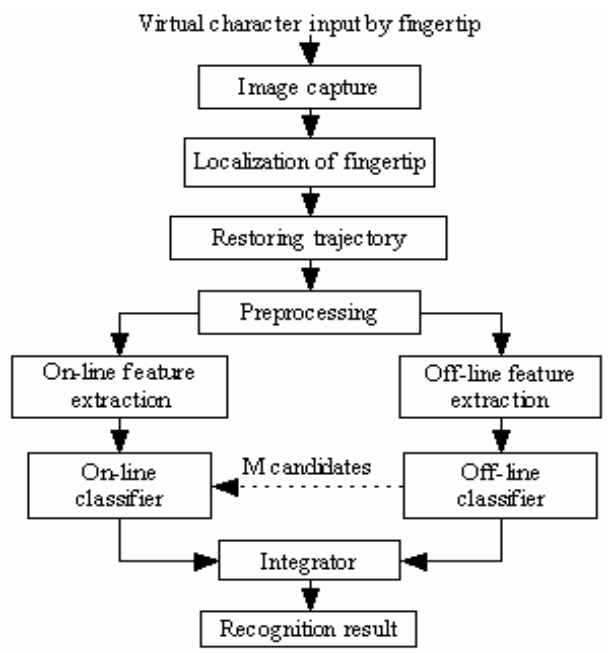

Fig. 2. Structure of the proposed recognition system

\section{Feature extraction}

\subsection{On-line feature extraction}

On-line recognition methods based on DTW usually use directional or positional features of feature points extracted along strokes [7][8]. We consider the writing direction as the main feature of strokes in a handwritten character. Positional feature is also used as a secondary feature in our on-line recognition approach. When constructing templates, only directional vectors are recorded. The positional features are dynamically regenerated from the directional vectors at run-time. The feature extraction of our on-line recognizer is discussed in more detail in our previous work [11].

\subsection{Off-line feature extraction}

Off-line recognition methods extract features from two dimensional character images. So the on-line characters represented by point sequences are converted to images before off-line feature extraction is performed. We use lines of three pixels width to connect successive points to construct the character image. A directional decomposition algorithm [12] is then applied to decompose the character image into four sub-patterns according to the contour-direction feature [10]. Experimental results have convinced us the grouping method (Fig. 3(b)) we used is more 
efficient and robust than the original one (Fig. 3(a)) proposed by Tseng et al. [10].

After a character image is decomposed into four directional sub-patterns, an elastic meshing method [9] is applied into the four sub-patterns respectively to partition each patterns into several small region (we call it mesh). We used $4 \times 2$ local elastic meshes to extract the cellular directional features. Examples of 8 $\times 8$ fixed meshing and $4 \times 2$ local elastic meshing methods are illustrated in Fig. 4. The distribution of black pixels in each mesh of four decomposed subpatterns is calculated and used as the off-line feature. Thus the feature vector is a 256-dimensional vector.

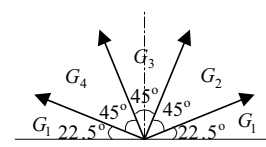

(a)

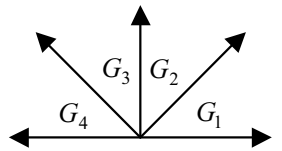

(b)
Fig. 3. Four direction region partition method. (a) original method [8]. (b) modified method.

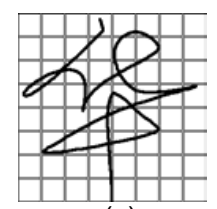

(a)

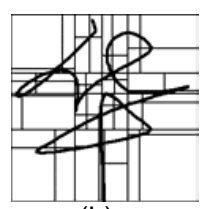

(b)
Fig. 4. (a) $8 \times 8$ fixed meshing method. (b). $4 \times 2$ local elastic meshing method.

\section{Classifier design}

\subsection{DTW based on-line classifier}

The classifier used in our on-line recognizer is based on prototype matching by dynamic time warping (DTW)[15]. By the matching score composed of directional and positional DTW global distance, prototypes are arranged in ascending order and the first one is regarded as the recognition result. It was discussed in [11].

\subsection{LDA based off-line classifier design}

The off-line feature of a handwritten character is a 256-dimensional vector. In order to improve the classification speed and recognition rate, linear discriminant analysis (LDA) [18][19], a well known dimensionality reduction technique, is employed to design the off-line classifier. We used a simple squared Euclidean distance classifier to classify the input handwritten character in a dimension reduced space which was obtained by LDA.

\section{Integration of on-line and off-line recognition}

In our recognition system, on-line and off-line recognition methods cope with different aspects of recognition problem in recognizing cursive one stroke characters: on-line recognition can tolerate large variations in stroke shape and position while off-line recognition is not sensitive to variations of stroke order. The complementary integration can provide the recognition system both advantages of the two recognition approaches.

After an input character is classified by on-line and off-line recognizer, two candidate sequences, on-line recognition result $A_{i}(i=1 . . M)$ and off-line recognition result $B_{j}(j=1 . . N)$, are produced. $M$ is a variable determined by a rejective score of on-line recognition, and $N$ is a constant. The positional score of each candidate is calculated as follows: by:

The positional score of on-line candidate is given

$$
S_{A i}=i \times \exp (1-i) \times C-P_{i},
$$

and the positional score of off-line candidate is given by:

$$
S_{B j}=j \times \exp (1-j) \times D+i^{\prime} \times \exp \left(1-i^{\prime}\right) \times C,
$$

$C$ and $D$ are the maximum score of on-line and offline candidates. $P_{i}$ is the penalty score corresponding to the $i$-th candidate's recognition score. $i^{\prime}$ is the index of the same candidate as $B_{j}$ in candidate sequence $A$. If there is no same candidate found in $A, i^{\prime}$ is set to a very large value.

The rejective score for obtaining $M$ is calculated by (3) and $M$ is obtained by (4).

$$
R_{i}=\left(W_{i}-W_{i-1}\right) /\left(W_{i-1}-W_{i-2}\right)(3)
$$

where $W_{i}$ is the $i$-th candidate's distance to the input.

$$
M=\arg \min \left(R_{i}<0.2\right)
$$

In the integration stage, candidates produced by online and off-line recognizer are put together and sorted in descending order according to the positional score given by eq. (1) and (2). The integrated candidate sequence is then produced by the sorted candidates. The following steps are carried out to perform the first candidate election:

Step1: If $\mathrm{A}_{0}$ and $\mathrm{B}_{0}$ are the same, then it is chosen as the first candidate.

Step2: If $\mathrm{A}_{0}$ is very confident and $\mathrm{B}_{0}$ is not confident, choose it from A.

Step3: If $\mathrm{B}_{0}$ is very confident, choose it. 
Step4: If $\mathrm{A}_{\mathrm{k}}=\mathrm{B}_{0}$ and $\mathrm{B}_{\mathrm{l}}=\mathrm{A}_{0}$, where $\mathrm{k}$ and 1 are less than 35 , if $\mathrm{k}<1$, then choose $\mathrm{B}_{0}$; vice versa.

Step5: If $A_{k}=B_{1}$ and $B_{1}=A_{1}$, where $k$ and 1 are less than 15 , if $\mathrm{k}<1$, then choose $\mathrm{B}_{0}$; vice versa.

If no condition is satisfied in all the steps above, the first candidate of the integrated and sorted candidate sequence will not be changed.

\section{Experimental results}

The experimental results for testing recognition efficiency of our on-line recognizer for English letters were given in [11]. As the recognition rate is good enough, we concentrated on designing our integrator for Chinese characters. Experiments in this section are focused on recognition of handwritten Chinese characters.

\subsection{Experimental data}

In experiments for testing recognition performance of Chinese cursive characters, 50 sets of handwritten Chinese characters (each set consists of 3755 categories of GB2312-80 level 1 Chinese characters, i.e. 187,750 characters in total) were used in experiments with strokes fully connected. We call this experimental data set ONHCC. They were written by 50 different individuals. And all the characters are written naturally with no constraint in stroke order, stroke number and writing style. Fig. 5 shows some samples in ONHCC.

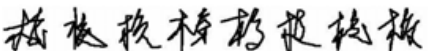

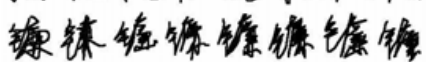

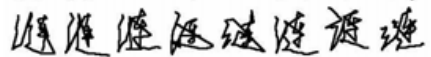

Fig. 5. Some on-line handwritten Chinese character samples, strokes of each character are connected.

\subsection{Experimental results on integration}

We performed some experiments to evaluate the performance of our on-line/off-line recognizer and the hybrid recognizer. The template of on-line recognizer was constructed by clustering from 40 sets of ONHCC, and each category was clustered to 2 prototypes on average. The off-line recognizer was also trained by these 40 sets. The other 10 sets in ONHCC were used to test the recognition efficiency. The experimental results are shown in Fig. 6.

Comparing the individual recognition rate of $80.6 \%$ given by off-line recognizer and $83.4 \%$ by on-line recognizer, the recognition rate after integration achieved $90.9 \%$, and the cumulative rates for the first 2 and 10 candidates were $95.2 \%$ and $98.6 \%$ respectively. The results were encouraging and proved that the integration is a success.

To demonstrate the recognition capability of our proposed hybrid system for virtual characters which may have strokes or radicals overwritten, some typical cursive character samples which can be correctly recognized are shown in Fig. 7.

Fig. 8 demonstrated several typical pairs of characters which are difficult to be distinguished. Note that some of these pairs are almost impossible to be distinguished when written in one-stroke style.

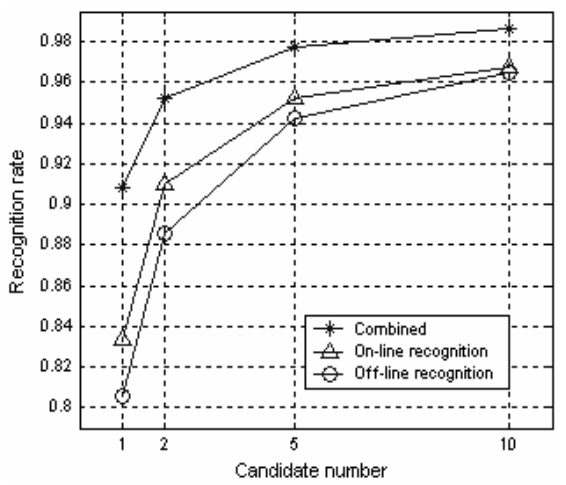

Fig. 6. Recognition rates of on-line/off-line recognizer and the combined recognizer.

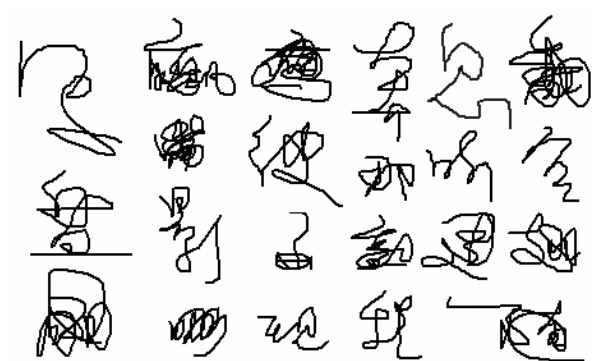

Fig. 7. Some typical one-stroke handwritten Chinese characters which can be correctly recognized by our hybrid recognition system.

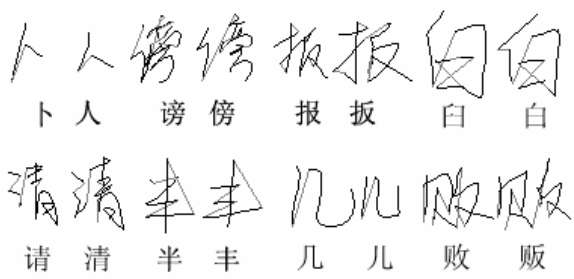

Fig. 8. Some typical pairs of characters which are difficult to be distinguished. 


\section{Conclusions}

In this paper, a novel hybrid one stroke cursive character recognition system is proposed. An on-line recognizer which can tolerate large variations in stroke shape and position and an off-line recognizer which is not sensitive to variations of stroke order are combined in this system. Benefit from the complementary integration which provides both advantages of the two recognizers, our recognition system can recognize stroke order free one stroke cursive characters. For character that is not written in one stroke style, we can link all its strokes together (virtual stroke) and then it can be also regarded as a kind of one stroke cursive character. From this viewpoint, our hybrid recognition approach is also stroke number free. Although the integration method we used was heuristic based, the experimental results proved the efficiency of such kind of hybrid recognition system. In future work, we'll compare different integration methods and report the results.

\section{Acknowledgement}

The paper is partly supported by the following research foundation: NSFC (No.60275005), GDNSF (No. 2003C50101, 04105938). The authors would like to thank Dr. Qiang Huo from the University of Hongkong for providing online handwritten Chinese character samples.

\section{References}

[1] R. Plamondon and S. N. Srihari, "On-line and Off-line Handwriting Recognition: A Comprehensive Survey," IEEE Transaction on Pattern Analysis and Machine Intelligence, vol. 22, no. 1, pp. 63-84, January 2000.

[2] C.-L. Liu, S. Jaeger, and M. Nakagawa, "Online Recognition of Chinese Characters: The State-of-the-Art," IEEE Transaction on PAMI, vol. 26, no. 2, pp. 198-213, February 2004.

[3] J.-Y. Zhang, X.-Q. Ding, C.-S. Liu, "Multi-scale feature extraction and nested-subset classifier design for high accuracy handwritten character recognition," Proc. 15th Int. Conf. on Pattern Recognition, pp. 581-584, 2000.

[4] K. Kiyota et al, "An improvement of on-line Japanese character recognition system for visually disabled persons," Proc. 14th Int. Conf. on Pattern Recognition, pp. 1752-1754, 1998.

[5] H. Shimodaira et al, "On-line Overlaid-Handwriting Recognition Based on Substroke HMMs," Proc. 7th Int. Conf. on Document Analysis and Recognition, pp. 1043-1047, 2003.

[6] "On-line cursive hangul recognition that uses DP matching to detect key segmentation points," Pattern Recognition, vol. 37, no. 11, pp. 2101-2112, 2004.
[7] Y.-T. Tsay, and W.-H. Tsai, "Attributed String Matching by Split-and-Merge for On-line Chinese Character Recognition," IEEE Transaction on PAMI, vol. 15, no. 2, pp. 180-185, February 1993.

[8] V. Vuori et al., "Experiments with Adaptation Strategies for a Prototype-Based Recognition System for Isolated Handwritten Characters," Int. J. Document Analysis and Recognition, vol. 3, no. 3, pp. 150-159, 2001.

[9] L.-W. Jin, G. Wei, "Handwritten Chinese Character Recognition with Directional Decomposition Cellular Features", Journal of Circuit, System and Computer, Vol.8, No.4, pp.517-524, 1999.

[10] Y.-H. Tseng, C.-C. Kuo, H.-J. Lee, "Speeding Up Chinese Recognition in an Automatic Document Reading System", Pattern Recognition, Vol. 31, No. 11, pp. 16011612, 1998.

[11] T. Long and L.-W. Jin, "One Stroke Cursive Character Recognition using Combination of Directional and Positional Features", Int. Conf. on Acoustics, Speech, and Signal Processing, (ICASSP '05), vol. 5, pp. 449-452, Philadelphia, USA, March 18-23, 2005.

[12] L.-W. Jin, J.-X. Yin, X. Gao, "Study of Several directional feature extraction methods with local elastic meshing technology for HCCR", Proceedings of the Sixth Int. Conference for Young Computer Scientist, pp232-236, Oct. 2001, Hang-Zhou, China.

[13] R.A. Fisher, "The Statistical Utilization of Multiple Measurements", Annals of Eugenics, vol. 8, pp. 376-386, 1938.

[14] R. Duda and P. Hart, Pattern Classification and Scene Analysis, John Wiley \& Sons, 1973.

[15] H. Sakoe and S. Chiba, "Dynamic programming algorithm optimization for spoken word recognition," IEEE Trans. Acoust., Speech, Signal Processing, vol. ASSP-26, pp. 43-49, Feb. 1978.

[16] S. Manke, U. Bodenhausen, "A connectionist recognizer for on-line cursive handwriting recognition," Int. Conf. on ICASSP '94, vol. 2, pp. 633-636, 1994.

[17] G. Seni, R.K. Srihari, N. Nasrabadi, "Large vocabulary recognition of on-line handwritten cursive words," IEEE Transaction on PAMI, vol. 18, no. 7, pp. 757-762, 1996.

[18] Munich, M.E.; Perona, P., "Visual input for pen-based computer," IEEE Transaction on PAMI, vol. 24 , no. 3, pp313-328, 2002.

[19] M. Lin, et al., "Hidden control neural network and HMM hybrid approach for on-line cursive handwriting recognition," Int. Conf. Neural Networks \& Signal Processing, pp. 236-239, 2003.

[20] H. Li, et al., "Writing style variation absorption for a hybrid Neuro-Markovian on-line handwriting recognition system," Int. Conf. on Knowledge-Based Intelligent Information Engineering System, pp. 1311-1315, 2002. 